Trauma Berufskrankh 2016 • [Suppl 1]: 18:S33-S38 DOI 10.1007/s10039-015-0099-0

Online publiziert: 18. November 2015

c) Springer-Verlag Berlin Heidelberg 2015

Christian Schoepp

Klinik für Arthroskopische Chirurgie, Sporttraumatologie und Sportmedizin,

BG Unfallklinik Duisburg, Duisburg

\title{
Rekonstruktion des vorderen Kreuzbandes
}

\section{Was ist gesichert?}

In den letzten Jahren nimmt die Anzahl der operativ behandelten vorderen Kreuzband (VKB)-Verletzungen drastisch zu. Für die USA wird hierbei ein Anstieg um $55 \%$ zwischen den Jahren 1994 und 2006 verzeichnet. Ursächlich für den Anstieg der operierten Fälle ist einerseits das veränderte Freizeitverhalten, andererseits aber auch die Zunahme der technischen Möglichkeiten in der Kreuzbandchirurgie [2]. Die Behandlungskosten je Kreuzbandersatzplastik sind immens und liegen bei über $80.000 €$. Dem operativen Eingriff schließt sich eine lange Rehabilitationsphase von bis zu 12 Monaten an. Shelbourne [14] beschreibt trotz der heutigen operativen Möglichkeiten eine Rerupturrate von $17 \%$. Trotz aller Bemühungen liegt die Arthroseprävalenz nach mehr als 10 Jahren (KellgrenLawrence $\geq 2$ ) bei isolierter VKBVerletzung bei $13 \%$, während die Prävalenz bei zusätzlichem Meniskusschaden mit $48 \%$ angegeben wird [10].

\section{Indikation zur Kreuzbandersatzplastik}

Die Frage, welcher Patient mit VKBRuptur operativ behandelt werden muss, wird weiterhin kontrovers diskutiert. Insbesondere bei isolierter VKB-Verletzung ohne initialen Meniskusriss besteht die Möglichkeit eines primär konservativen Behandlungsansatzes mit gezielter Rehabilitation. In einer vielfach zitierten Studie konnten Frobell et al. [4] zeigen, dass bei prospektiv randomisiertem Studiendesign nach 24 bzw. 60 Monaten die Gruppe der zeitnah nach dem Unfall operierten Patienten vergleichbar gute
Ergebnisse zeigte wie die Gruppe derjenigen Patienten, die primär konservativ rehabilitiert wurden. Der Studienarm der konservativ anbehandelten Patienten wies jedoch die Besonderheit auf, dass sich die Studienteilnehmer zu jedem Zeitpunkt bei subjektiver Instabilität auch operativ stabilisieren lassen konnten. Innerhalb von 24 Monaten ließen sich in dieser Art 23 von 59 Patienten nach konservativer Anbehandlung im weiteren Follow-up operieren. Von diesen 23 Patienten wiesen intraoperativ 11 Patienten neben der Kreuzbandverletzung einen zusätzlichen Meniskusschaden auf, der im initialen, zeitnah nach dem Unfall durchgeführten MRT noch nicht sichtbar war. Hieraus kann geschlussfolgert werden, dass die trotz Rehabilitation persistierende Instabilität offensichtlich in fast $50 \%$ der Fälle sekundäre Meniskusschäden nach sich zog [13]. Aus diesem Studiendesign und den berichteten Ergebnissen kann abgeleitet werden, dass hinsichtlich Indikationsstellung und Operationszeitpunkt eine individuelle Herangehensweise zielführend ist. Von unterschiedlicher Seite wurden diesbezüglich in der Vergangenheit Indikatoren beschrieben, die als Entscheidungshilfe für bzw. gegen eine operative Intervention herangezogen werden sollten. Hierzu zählen zweifelsfrei die initiale Begleitverletzung, die bevorzugte Sportart, die grundsätzliche sportliche Aktivität, das subjektive Instabilitätsempfinden und insbesondere die objektivierbare Instabilität, nachweisbar in spezifischen Tests [3].

\section{Operationszeitpunkt}

Untersuchungen aus den 1990er-Jahren legten die Vermutung nahe, dass das Risiko einer Arthrofibrose bei zeitnah nach Trauma durchgeführter Kreuzbandersatzplastik erhöht sei. Diese Studien beinhalteten jedoch zum Teil noch offene Operationstechniken und eine sehr restriktive, zum Teil durch Gipsruhigstellung unterstützte Nachbehandlung [15].

In einer jüngeren Metaanalyse aus dem Jahr 2013 unter Einschleusung von mehr als 900 Patienten zeigte sich, dass unabhängig von der Verwendung von Hamstring-Transplantaten oder BonePatella-Tendon-Bone-Transplantaten kein erhöhtes Arthrofibroserisiko gesehen wurde, wenn innerhalb der ersten, zweiten oder auch dritten Woche nach Trauma operiert wurde [7]. Die aktuelle Studienlage lässt daher die Schlussfolgerung zu, dass der korrekte Operationszeitpunkt letztendlich individuell, insbesondere auch abhängig von den Begleitverletzungen $\mathrm{zu}$ sehen ist. Dennoch stellt die posttraumatische/postoperative Arthrofibrose eine Komplikation dar, die es zu vermeiden gilt. Der reizfreie Gelenkzustand ist daher auch weiterhin erstrebenswert.

\section{Transplantatwahl und Transplantateigenschaften}

Die Semitendinosussehne allein oder in Kombination mit der Gracilissehne ist das zum gegenwärtigen Zeitpunkt am häufigsten verwendete Sehnentransplantat für die VKB-Ersatzplastik. Ein zentraler Quadrizepssehnenstreifen mit 


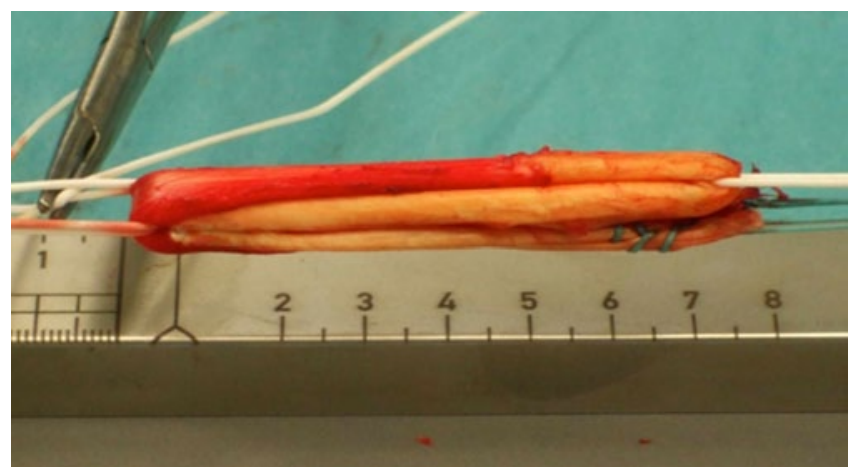

Abb. 1 A Sehnenpräparation

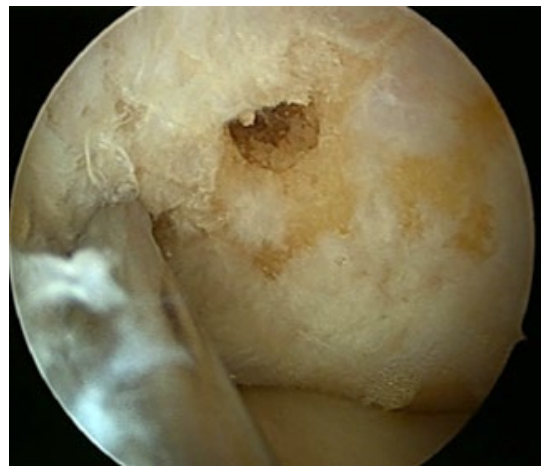

Abb. 3 A Medialer Portalblick

oder ohne Knochenblock erfreut sich in jüngster Zeit immer größerer Beliebtheit, und dies nicht nur für den Revisionsfall, sondern auch für den Primäreinsatz. Daneben hat sich das mittlere Patellarsehnendrittel mit 1 oder 2 Knochenblöcken über viele Jahre als Kreuzbandtransplantat bewährt. Die Auswahl des geeigneten Sehnentransplantates hängt vom Patientenalter, von der Konstitution des Patienten, nicht zuletzt aber auch von der Vorliebe des Operateurs ab. Grundsätzlich gilt, dass ein Kreuzbandoperateur mindestens 2 alternative Verfahren sicher beherrschen sollte. Hinsichtlich des verwendeten Transplantatdurchmessers gibt es insbesondere bei Verwendung der Semitendinosussehne mit oder ohne Gracilissehne erhebliche Variabilitäten. So konnte jüngst in einer biomechanischen Studie gezeigt werden, dass die Reißfestigkeit des verwendeten Konstruktes bei einer Transplantatdurchmesserzunahme von 7 auf $8 \mathrm{~mm}$ um $20 \%$ wächst, bei einer Transplantatdurchmesserzunahme von 7 auf 9 mm sogar um 34\% [1]. Darüber hinaus scheint der Transplantatdurchmesser auch einen unmittelbaren Einfluss auf die Rerupturrate zu haben [8].

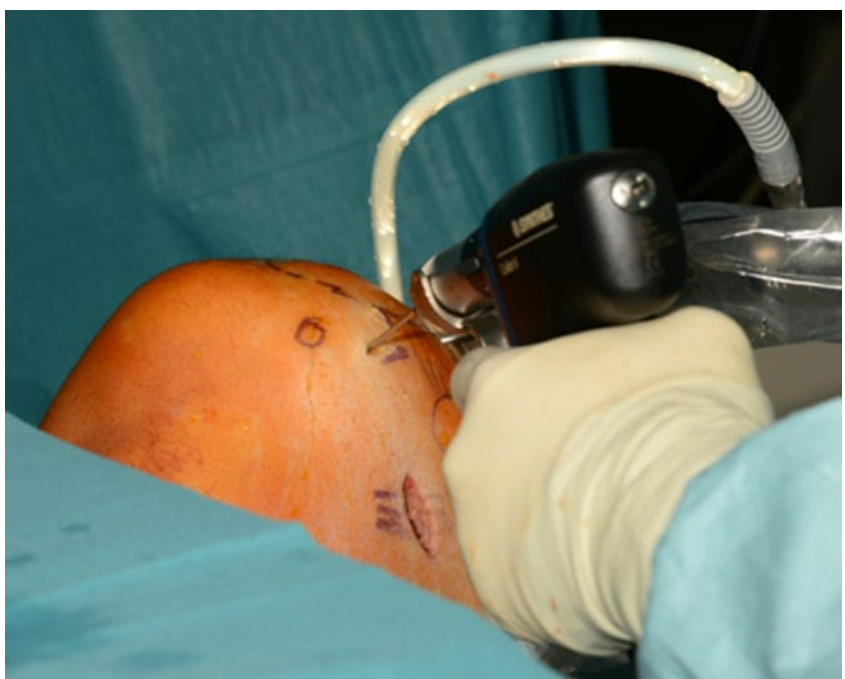

Abb. 2 А Femorale Bohrkanalanlage

Um den gewünschten Transplantatdurchmesser $\mathrm{zu}$ erreichen, ist insbesondere bei Verwendung der Hamstring-Sehnen ein differenziertes Vorgehen notwendig. So muss letztendlich individuell und in Abhängigkeit der Sehnenqualität entschieden werden, ob ein 3-fach-, 4-fach-, 5-fach oder sogar 6-fach-Strang letztendlich den geeigneten Transplantatdurchmesser erbringt (• Abb. 1).

Die Definition eines Mindestdurchmessers für die individuelle Kreuzbandchirurgie macht keinen Sinn. Dennoch gibt es Studien, die belegen, dass die Revisionsrate bei Transplantatdurchmessern von bis zu $8 \mathrm{~mm}$ deutlich höher ist als bei Transplantatdurchmessern von mehr als $8 \mathrm{~mm}$ [8].

\section{Bohrkanalanlage}

Die Anlage des tibialen Bohrkanals richtet sich idealerweise nach dem originären VKB-Stumpf, sofern dieser im Rahmen der Kreuzbandersatzplastik noch vorhanden ist. Eine wesentliche Landmarke stellt darüber das Außenmeniskusvorderhorn dar. Eine unmittelbare Anlehnung des tibialen Bohrkanals an den intraartikulären Verlauf des hinteren Kreuzbandes führt jedoch zu einer zu weit posterioren tibialen Bohrkanalanlage.

Die Anlage des femoralen Bohrkanals erfolgt in der älteren Technik transtibial. Das bedeutet, dass die Positionierung des femoralen Bohrkanals unmittelbar von der Qualität und Positionierung des tibialen Bohrkanals abhängig ist. Um dieses Abhängigkeitsverhältnis aufzubrechen, wurde in jüngerer Vergangenheit die Portaltechnik entwickelt. Hierbei wird über ein tiefes anteromediales Portal in $110-120^{\circ}$ Kniegelenkbeugung unabhängig vom tibialen Bohrkanal ein femoraler Tunnel angelegt (• Abb. 2).

Da die Übersicht im Kniegelenk bei starker Beugung unter Umständen limitiert ist, hat sich der mediale Portalblick über ein hohes mediales Portal als hilfreich erwiesen, um die Lage des femoralen VKB-Footprints adäquat zu überprüfen (- Abb. 3). Durch verschiedene Studien ist mittlerweile bewiesen worden, dass das femorale Insertionsgebiet des VKB bei transtibialer Bohrkanalanlagetechnik sehr viel seltener erreicht wird als bei der medialen Portaltechnik. Der theoretische Nutzen der unabhängig voneinander angelegten Bohrkanäle findet in einer groß angelegten Studie mit mehr als 8000 Patienten (Dänisches Kreuzbandregister) allerdings kein klinisches Korrelat. So war das Risiko für eine Revision nach anteromedialer femoraler Tunnelanlage signifikant höher als bei transtibialer Tunnelanlage [12]. Unter Umständen ist die höhere Revisionsrate der jüngeren Technik und somit de facto einer Lernkurve geschuldet. Weitere Studien müssen zeigen, ob die mittlerweile empfohlene anteromediale femorale Tunnelanlage dauerhaft den gewünschten klinischen Erfolg bringen wird. 


\section{Einzelbündel- oder Doppelbündelrekonstruktion}

Da das VKB aus mindestens 2 anatomischen Bündeln besteht (anteromediales und posterolaterales Bündel), haben sich in der Vergangenheit viele Arbeitsgruppen mit der Doppelbündelrekonstruktion des VKB beschäftigt. In einer kürzlich erschienenen Metaanalyse zeigte sich nach Doppelbündelrekonstruktion ein signifikant besserer objektivierbarer Wert für die a.p.-Translation und den Pivot-ShiftTest. In den subjektiven Scores fand sich jedoch kein signifikanter Unterschied [9]. Vergleichbar waren zwischen Einzelbündel- und Doppelrekonstruktion sowohl die Rerupturrate als auch die perioperative Komplikationsrate.

Zusammenfassend kann aufgrund der aktuellen Studienlage die Doppelbündelrekonstruktion des VKB nicht als chirurgischer Goldstandard definiert werden. Vielmehr ist die Doppelbündelrekonstruktion individuellen Einzelfällen vorbehalten.

\section{Transplantatfixierung}

Grundsätzlich unterscheidet man verschiedene Fixationsprinzipien. Unterschieden wird die gelenknahe von der gelenkfernen Fixation. Die klassische gelenknahe Fixation ist die Interferenzschraubenfixierung mit degradierbaren oder metallenen Implantaten. Abzugrenzen hiervon sind die gelenkfernen extrakortikalen Fixationstechniken, bei denen das Kreuzbandtransplantat über Fadenmaterial gelenkfern fixiert wird.

Als Hybridfixation wird die Kombination aus gelenkferner und gelenknaher Fixation bezeichnet. Dieses Prinzip kann sowohl auf der femoralen als auch auf der tibialen Seite angewendet werden (• Abb. 4).

In letzter Zeit erfreuen sich implantatfreie Fixationstechniken einer immer höheren Beliebtheit. Hier bieten sich insbesondere Transplantate mit 1 oder 2 Knochenblöcken an (• Abb. 5).

Trauma Berufskrankh 2016 · [Suppl 1]: 18:S33-S38 DOI 10.1007/s10039-015-0099-0

๑) Springer-Verlag Berlin Heidelberg 2015

\section{Schoepp}

\section{Rekonstruktion des vorderen Kreuzbandes. Was ist gesichert?}

\section{Zusammenfassung}

Die Behandlung der vorderen Kreuzband (VKB)-Ruptur hat für Klinikärzte wie für niedergelassene Kollegen einen hohen Stellenwert. Bis zum heutigen Tag sind viele Fragen zum Outcome, aber auch zur technischen Durchführung einer VKBErsatzplastik nicht abschließend geklärt. Dass die stabilisierende Operation eine Arthrose verhindern oder zumindest zeitlich aufhalten kann, ist bis heute nicht erwiesen. Allerdings ist bekannt, dass ein instabiles Kniegelenk Sekundärschäden z. B. in Form von Meniskusrissen nach sich zieht. Der Operationszeitpunkt ist abhängig von Begleitverletzungen und dem tatsächlichen Ausmaß der Instabilität. Als körpereigenes Transplantat stehen die Semitendinosussehne (mit oder ohne Gracilissehne), ein zentraler Patellarsehnenstreifen mit Knochenblöcken oder ein zentraler Quadrizepssehnenstreifen mit und ohne Knochenblock zur Verfügung. Neben der Transplantatwahl hat auch die Verwendung eines adäquaten Sehnendurch- messers eine wesentliche Bedeutung. In der Technik der Bohrkanalanlage zeichnet sich eine Weiterentwicklung ab. Ziel ist die anatomische Bohrkanalanlage. Die Doppelbündelrekonstruktion trägt dazu bei, die Anatomie des Originalbandes besser nachzuempfinden. Der wissenschaftliche Ansatz hat jedoch zu keiner richtungsweisenden Ergebnisverbesserung beigetragen. Die Fixationstechniken werden nach gelenkferner, gelenknaher und implantatfreier Fixation unterschieden. Echte Rerupturen sind seltener als ein Transplantatversagen infolge operationstechnischer Fehler im Zusammenhang mit der Tunnelanlage oder Transplantatfixation. Auch übersehene Instabilitäten können einen negativen Einfluss auf das Outcome nach VKB-Rekonstruktion haben.

\section{Schlüsselwörter \\ Kreuzbandruptur - Operation - Arthrose . \\ Transplantat · Fixation}

\section{Reconstruction of the anterior cruciate ligament. What is sure?}

\section{Abstract}

The treatment of ruptures of the anterior cruciate ligament $(\mathrm{ACL})$ plays an essential role for both clinicians and resident physicians. To date many questions regarding the outcome as well as ACL reconstruction techniques have not yet been conclusively clarified. Whether reconstruction of the ACL protects the knee from osteoarthritis is still unproven; however, it is well known that an unstable knee joint is more vulnerable to secondary injuries, such as meniscal tears. Thus, early $\mathrm{ACL}$ reconstruction is recommended to minimize the risk of these secondary injuries. Three alternative sources of material for autologous ACL reconstruction are commonly utilized. An accessory hamstring (i.e. semitendinosus tendon with or without the gracilis tendon), a central strip of the patellar tendon with bone blocks and a central strip of the quadriceps tendon with or without bone block are the most common donor tissues used in autografts. Besides selection of the type of graft, the tendon diame- ter also plays a crucial role. Some progress has recently been made with respect to tunnel placement. The aim is to find an anatomical tunnel position. Reconstruction of both the anteromedial and the posterolateral $\mathrm{ACL}$ bundles helps to rebuild the anatomy of the original ACL; however, scientifically this approach did not lead to any improvement in the results. For fixation techniques a differentiation is made between aperture, extracortical and implant-free fixation. Generally, reruptures are less common than revisions as a result of graft ruptures due to technical mistakes during surgery. The most common mistakes concern tunnel placement and graft fixation. Also overlooked instability can have a negative influence on the outcome of ACL reconstruction.

\section{Keywords}

Cruciate ligament rupture - Transplant .

Operation · Arthrosis · Fixation

\section{Versagensursachen}

Echte Rerupturen nach VKBErsatzplastik treten in einer Häufig- keit von 3,6-10\% auf. Sehr viel häufiger sind jedoch operationstechnische Fehler, die in einer Größenordnung von mehr 

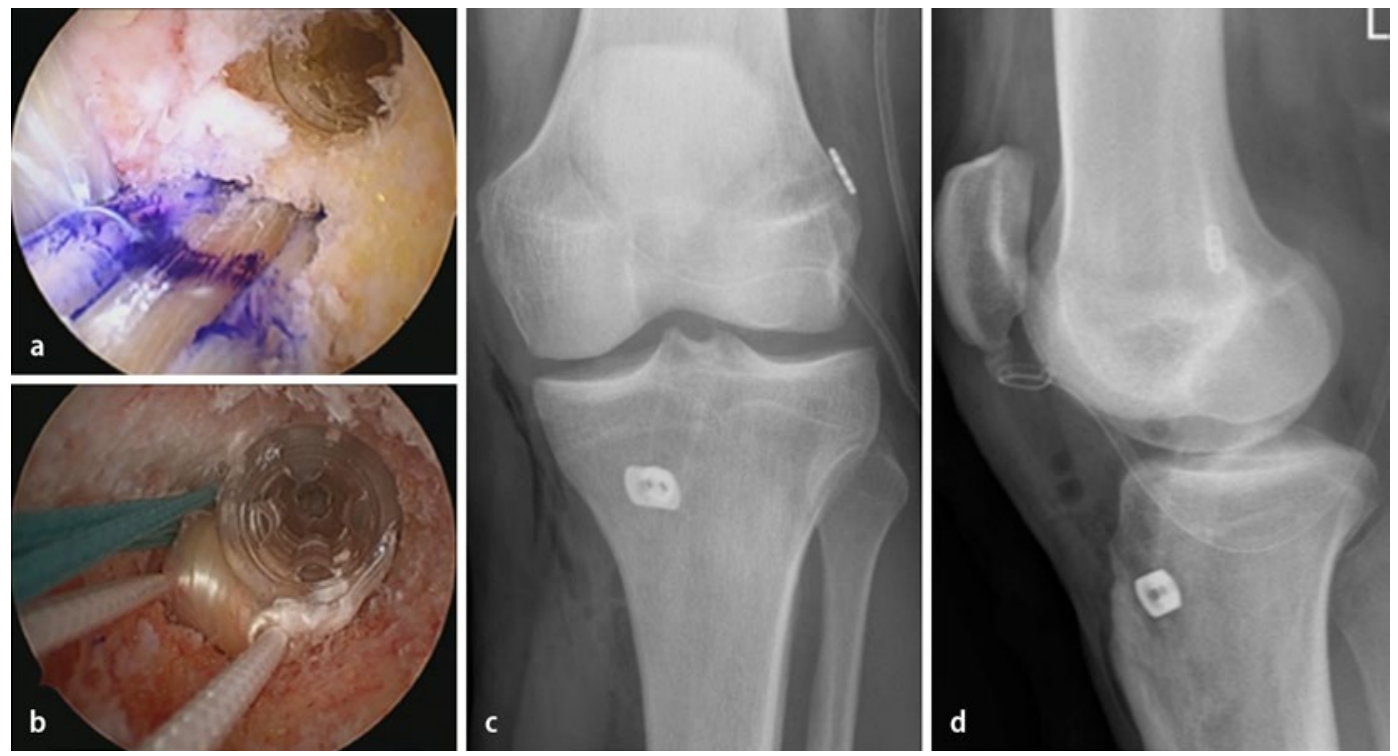

Abb. $4<$ Hybridfixation. a Femorale Interferenzschraube (Bone-WedgeTechnik). b Tibiale Interferenzschraube. $\mathbf{c}$, $\mathbf{d}$ a.p.und seitliches Röntgenbild postoperativ mit femoraler und extrakortikaler Fixierung (gleicher Patient wie in Teilabbildung a und $b$ )
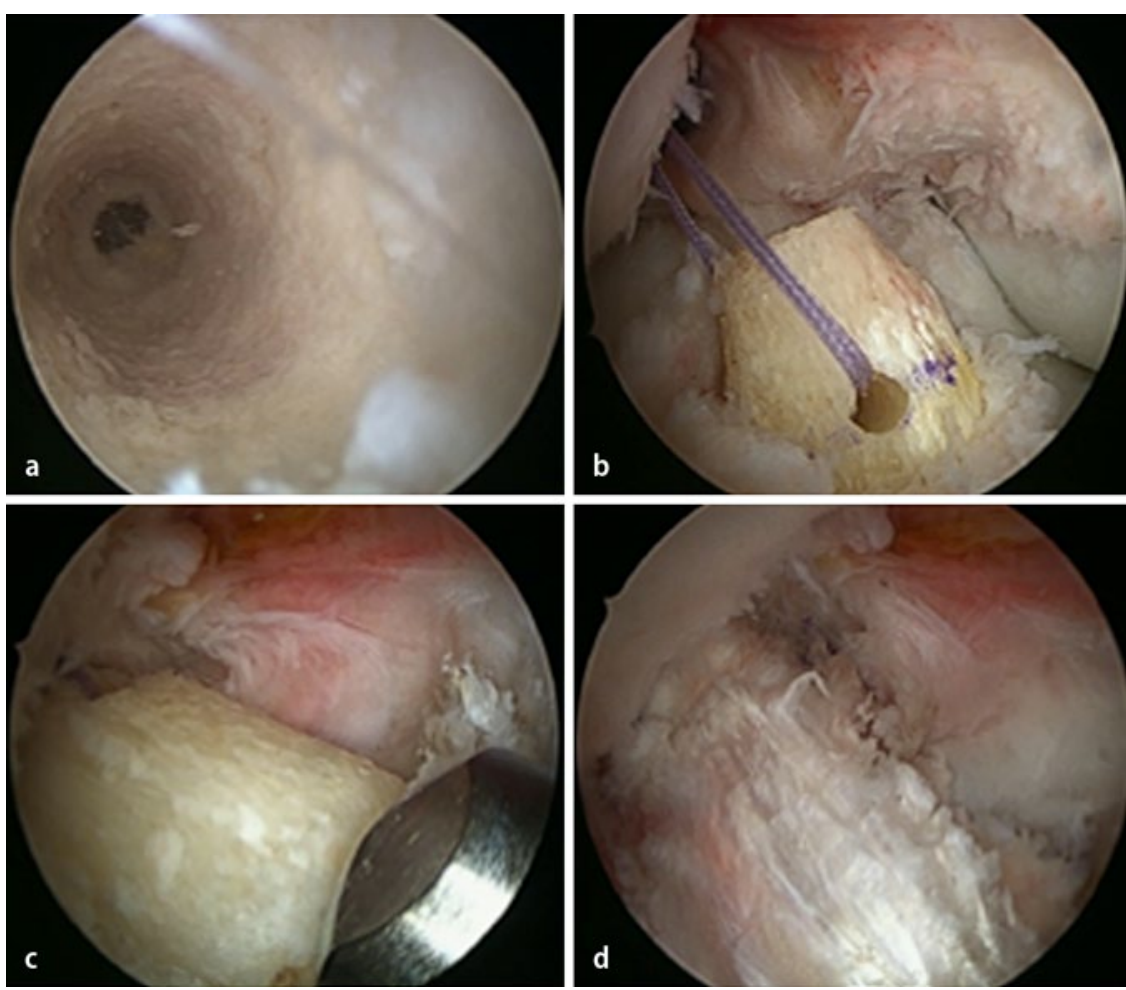

Abb. $5 \Delta$ Implantatfreie Fixation. a Femorale Tunnelanlage. b Einzug des Knochenblocks in das Gelenk. c Einstößeln des Knochenblocks in den femoralen Bohrkanal ("pressfit"). d Quadrizepssehnentransplantat in situ nach femoraler Fixation des Knochenblocks

als $15 \% \mathrm{zu}$ einem Transplantatversagen führen [5].

Typische Fehlerquellen sind:

- fehlerhafte Tunnelplatzierung,

- fehlerhafte Fixierung,

- übersehene Begleitinstabilitäten.

Fehlerhafte Tunnelplatzierungen münden zum Teil in eine groteske Bild- morphologie (• Abb. 6). Während die femorale Bohrkanalfehllage in aller Regel in derartigen Fällen unproblematisch im Rahmen eines einzeitigen Revisionskreuzbandersatzes korrigiert werden kann, verursachen massive Bohrkanalaufweitungen insbesondere auf der tibialen Seite häufig die Notwendigkeit eines zweizeitigen Vorgehens mit zwischenzeitlicher Bohr- kanalrevision unter Verwendung von körpereigener Beckenkammspongiosa oder alternativ Allografts.

Übersehene Begleitinstabilitäten können sowohl das hintere Kreuzband als auch die peripheren Kniegelenkstabilisatoren auf der medialen oder lateralen Seite betreffen.

Neuere anatomische und biomechanische Studien haben aufgezeigt, dass die fehlende Mitbehandlung von knöchernen und ligamentären Kapselbandverletzungen auf der Lateralseite [anterolaterales Ligament (ALL)] persistierende Instabilitäten begünstigen kann [11]. In diesem Zusammenhang kommt der Segond-Fraktur in jüngster Zeit wieder vermehrt Aufmerksamkeit zu. Sie ist ein klarer Hinweis auf das Vorliegen von Zusatzverletzungen am anterolateralen Kapselbandapparat, ohne dass sich hieraus per se eine Mitbehandlungsnotwendigkeit ergibt. Ein klarer Algorithmus, wann eine periphere Begleitinstabilität operativ mit zu adressieren ist und wann nicht, fehlt bis heute.

\section{Fazit für die Praxis}

- Die Verletzung des VKB bedarf hinsichtlich des therapeutischen Algorithmus einer differenzierten Indikationsstellung.

- Ziel muss es sein, das subjektiv und objektiv instabile Knie zeitnah zu stabilisieren. Allerdings kann trotz stabilisierender Maßnahmen die 

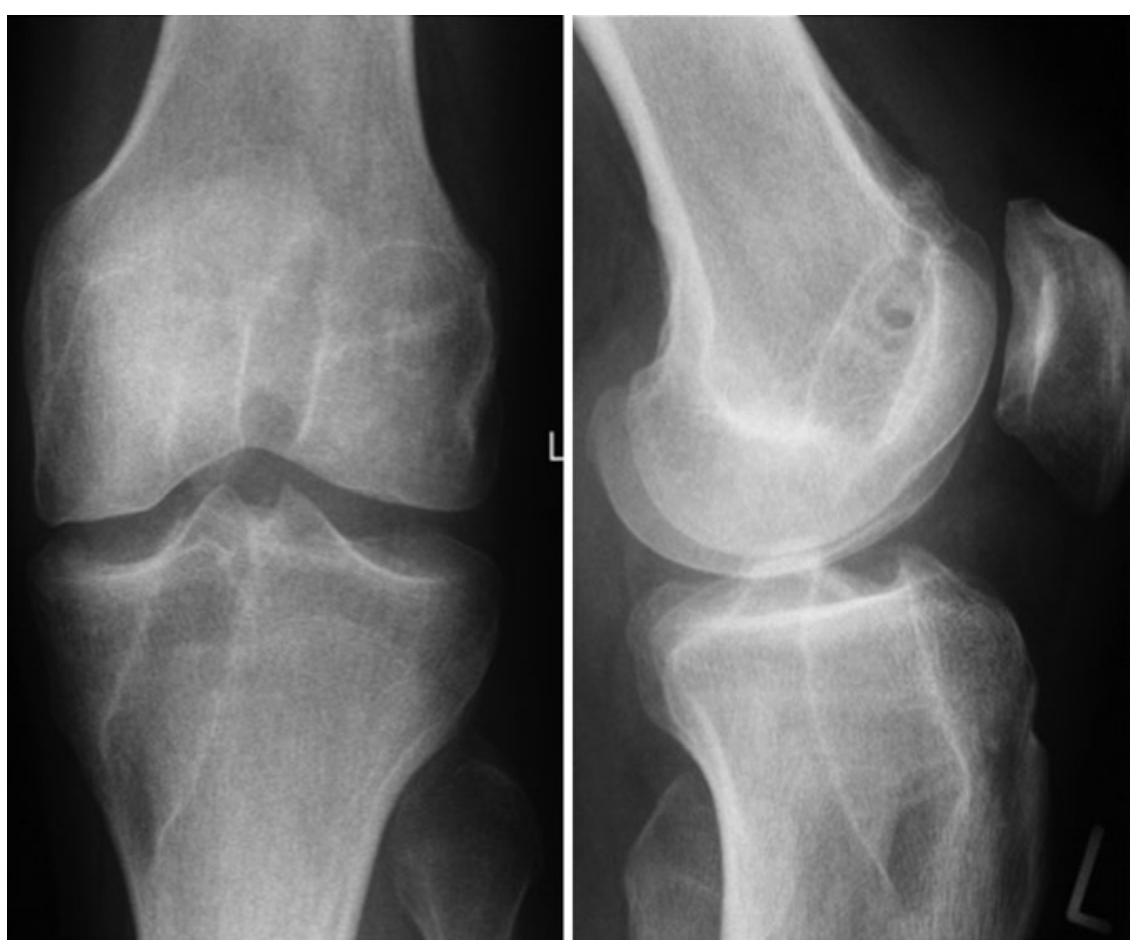

Abb. $6 \Delta$ Tunnelfehlplatzierung

Arthrose nach heutigem Kenntnisstand durch eine stabilisierende Kreuzbandersatzplastik nicht verhindert werden, insbesondere bei begleitenden Meniskusverletzungen.

- Hinsichtlich der Transplantateigenschaften ist darauf zu achten, dass unabhängig vom gewählten Transplantat ein adäquater Transplantatdurchmesser anzustreben ist.

- Ob die femorale Bohrkanalanlage in Portaltechnik neben den theoretischen Vorteilen auch tatsächlich für das klinische Outcome Vorteile bringt, muss anhand weiterer Studien noch belegt werden.

- Belegt ist die Einzelbündelrekonstruktion des VKB als Goldstandard, während die Doppelbündelrekonstruktion eher Ausnahmefällen zukommt.

- Hinsichtlich der Fixationstechniken gibt es keine überzeugende Tendenz, die gelenkferne oder gelenknahe Fixationstechniken mit sich bringen.

- Wichtig ist das Erkennen und Mitbehandeln von Begleitinstabilitäten, um eine sekundäre Transplantatinsuffizienz zu verhindern.

- Essenziell ist eine gute Ausbildung des Operateurs, um die noch immer
2. Buller LT, Best MJ, Baraga MG, Kaplan LD (2015) Trends in anterior cruciate ligament reconstruction in the United States. Orthop J Sports Med 3(1). http://doi.org/10.1177/2325967114563664

3. Fitzgerald GK, Axe MJ, Snyder-Mackler L (2000) A decision-making scheme for returning patients to high-level activity with nonoperative treatment after anterior cruciate ligament rupture. Knee Surg Sports Traumatol Arthrosc 8(2):76-82. http://doi. org/10.1007/s001670050190

4. Frobell RB, Roos EM, Roos HP, Ranstam J, Lohmander LS (2010) A randomized trial of treatment for acute anterior cruciate ligament tears. N Engl J Med 363(4):331-342. http://doi. org/10.1056/NEJMoa0907797

5. George MS, Dunn WR, Spindler KP (2006) Current concepts review: revision anterior cruciate ligament reconstruction. Am J Sports Med 34(12):2026-2037. http://doi. org/10.1177/0363546506295026

6. Bauer G, Buchner M, Schmitt H, Engelhardt M, Krüger-Franke M, Benedetto KP, Mayr H, Ellermann A, Miltner O (2010) Vorderes Kreuzband-OP-Indikation. GOTS Expertenmeeting: Vorderes Kreuzband: $S$ 53-58

7. Kwok CS, Harrison T, Servant C (2013) The optimal timing for anterior cruciate ligament reconstruction with respect to the risk of postoperative stiffness. Arthroscopy 29(3):556-565. http://doi.org/10.1016/j.arthro.2012.09.005

8. Mariscalco MW, Flanigan DC, Mitchell J, Pedroza AD, Jones MH, Andrish JT, Magnussen RA (2013) The influence of hamstring autograft size on patient-reported outcomes and risk of revision after anterior cruciate ligament reconstruction: a Multicenter Orthopaedic Outcomes Network (MOON) Cohort Study. Arthroscopy 29(12):19481953. http://doi.org/10.1016/j.arthro.2013.08.025

9. Mascarenhas R, Cvetanovich GL, Sayegh ET, Verma NN, Cole BJ, Bush-Joseph C, Bach BR (2015) Does double-bundle anterior cruciate ligament reconstruction improve postoperative knee stability compared with single-bundle techniques? A systematic review of overlapping meta-analyses. Arthroscopy 31(6):1185-1196. http://doi.org/ 10.1016/j.arthro.2014.11.014

10. Øiestad BE, Engebretsen L, Storheim K, Risberg MA (2009) Knee osteoarthritis after anterior cruciate ligament injury: a systematic review. Am J Sports Med 37(7):1434-1443. http://doi. org/10.1177/0363546509338827

11. Pomajzl R, Maerz T, Shams C, Guettler J, Bicos J (2015) A review of the anterolateral ligament of the knee: current knowledge regarding its incidence, anatomy, biomechanics, and surgical dissection. Arthroscopy 31(3):583-591. http://doi. org/10.1016/j.arthro.2014.09.010

12. Rahr-Wagner $L$, Thillemann TM, Pedersen AB, Lind $M$ (2014) Comparison of hamstring tendon and patellar tendon grafts in anterior cruciate ligament reconstruction in a nationwide populationbased cohort study: results from the danish registry of knee ligament reconstruction. Am J Sports Med 42(2):278-284. http://doi. org/10.1177/0363546513509220

13. Richmond JC, Lubowitz JH, Poehling GG (2011) Prompt operative intervention reduces long-term osteoarthritis after knee anterior cruciate ligament tear. Arthroscopy 27(2):149-152. http://doi.org/ 10.1016/j.arthro.2010.11.060 
14. Shelbourne KD, Gray T, Haro M (2009) Incidence of subsequent injury to either knee within 5 years after anterior cruciate ligament reconstruction with patellar tendon autograft. Am J Sports Med 37(2):246-251. http://doi. org/10.1177/0363546508325665

15. Shelbourne KD, Wilckens JH, Mollabashy A, DeCarlo M (1991) Arthrofibrosis in acute anterior cruciate ligament reconstruction. The effect of timing of reconstruction and rehabilitation. Am J Sports Med 19(4):332-336 